\title{
A Study of the Optical and Radiation Damage Properties of Lead Tungstate Crystals
}

\author{
C.L.Woody, J.A.Kierstead, S.P.Stoll \\ Brookhaven National Laboratory ${ }^{1}$ \\ R.Y.Zhu, D.A.Ma, H.B.Newman \\ California Institute of Technology ${ }^{2}$
}

\begin{abstract}
A study has been made of the optical and radiation damage properties of undoped and niobium doped lead tungstate crystals. Data were obtained on the optical absorbance, the intensity and decay time of the scintillation light output, and the radioluminescence and photoluminescence emission spectra. Radiation damage was studied in several undoped and niobium doped samples using ${ }^{60} \mathrm{Co}$ gamma ray irradiation. The change in optical absorption and observed scintillation light output was measured as a function of dose up to total cumulative doses on the order of $800 \mathrm{krad}$. The radiation induced phosphorescence and thermoluminescence was also measured, as well as recovery from damage by optical bleaching and thermal annealing. An investigation was also made to determine trace element impurities in several samples.
\end{abstract}

\section{INTRODUCTION}

Lead tungstate $\left(\mathrm{PbWO}_{4}\right)$ is a new scintillating material which is of great interest for use in high energy electromagnetic calorimeters [1-2]. It has a very high density ( $\rho$ $\left.=8.3 \mathrm{~g} / \mathrm{cm}^{3}\right)$, short radiation length $\left(\mathrm{X}_{0}=0.9 \mathrm{~cm}\right)$ and small Moliere radius $\left(\mathrm{R}_{M} \approx 2 \mathrm{~cm}\right)$, and has a scintillation light output which peaks between $450-550 \mathrm{~nm}$ with a fast component decay time in the range from 2-20 ns. It is presently being considered for use in two large, high resolution electromagnetic calorimeters, one for the CMS experiment and the other for the ALICE experiment, at the Large Hadron Collider at CERN. In order to meet the stringent demands of these two experiments, the crystals are required to be of high purity, produce uniform light output, and, in the case of CMS, be resistant to radiation damage up to several megarads.

We have carried out a study of the optical and radiation damage properties of a number of undoped and niobium doped crystals produced in Bogoroditsk, Russia [3]. A total of seven samples, measuring typically $2 \times 2 \times 21 \mathrm{~cm}^{3}$,

\footnotetext{
${ }^{1}$ Supported in part under Department of Energy Contract No. DE-AC02-CH7600016.

${ }^{2}$ Supported in part under Department of Energy Contract No. DE-FG03-92-ER40701.
}

were measured in all. In this paper, we will mainly discuss the results for two particular samples (number 767 and 768), both of which are $\mathrm{Nb}$ doped, and were part of a group of crystals prepared for study by CMS. These two samples, which were grown under similar conditions, showed very different properties, particularly with respect to radiation damage. Other samples, both undoped and $\mathrm{Nb}$ doped, have shown similar behavior, some exhibiting good radiation hardness and others not. We will compare a number of differences in samples 767 and 768 in order to attempt to identify which of these properties could be relevant in determining the important optical and radiation damage properties of $\mathrm{PbWO}_{4}$. Additional information on an undoped sample can be found in ref. [4].

\section{OPTICAL AND RADIATION DAMAGE PROPERTIES}

The initial (pre-irradiation) transmission spectra for samples 767 and 768 , measured down the longitudinal axis of the crystals using a Hitachi 3210 spectrophotometer, are shown in Figure 1. At longer wavelengths, the transmission of sample 767 is considerably less than for 768. This is because of a small ( $\sim 2 \mathrm{~cm}$ long) region of inhomogeneity near the center of the crystal which produced scattering and absorption near one end. At shorter wavelengths, the transmission of both samples extends down to approximately $350 \mathrm{~nm}$. Above the absorption edge, the transmission for sample 767 increases sharply and shows a distinct absorption band around $430 \mathrm{~nm}$, while for sample 768 , the transmission increases more slowing and indicates a number of possible broad absorption bands in this region.

The spectral light output of both samples was compared by measuring the radioluminescence and photoluminescence emission spectra. The radioluminescence was measured using ${ }^{60} \mathrm{Co}$ gamma ray excitation at the Gamma Ray Irradiation Facility at Brookhaven National Lab [5]. The photoluminescence was measured using an Oriel 77250 monochromator and an EMI 9659 phototube with UV excitation at a wavelength of $315 \mathrm{~nm}$. For sample 767 , the photoluminescence was also measured for other excitation wavelengths from 250-350 nm, and no dependence of the emission spectrum on the excitation 
1586
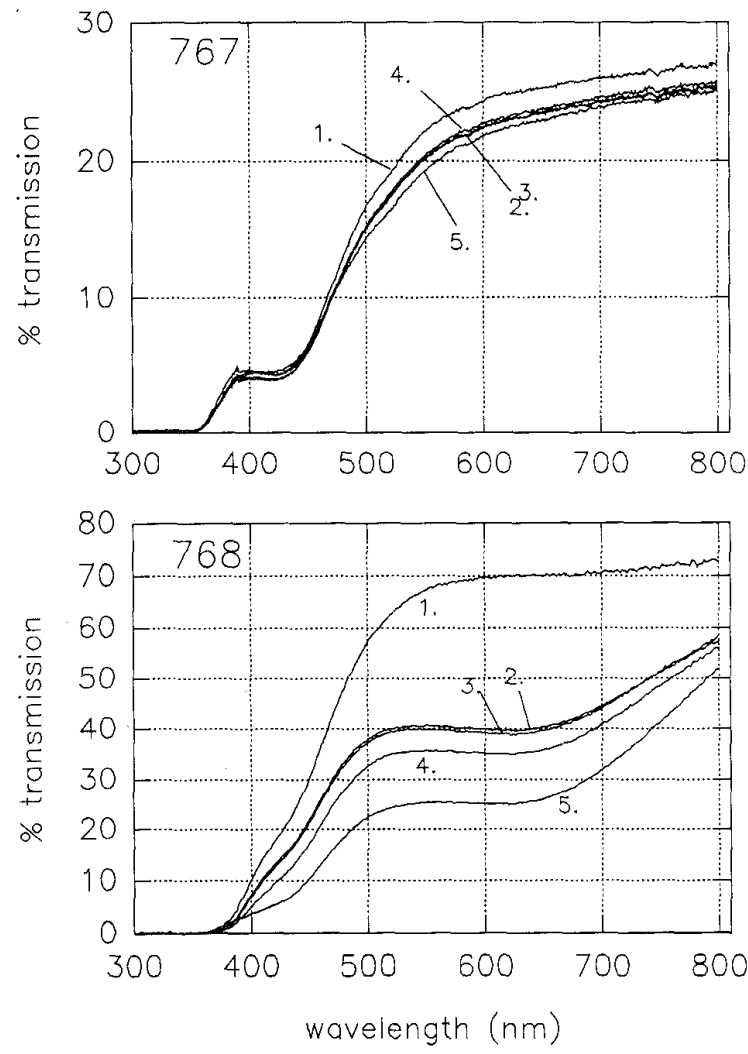

Figure 1: Longitudinal transmission spectra as a function of radiation dose for samples 767 and 768. Cumulative doses for 767 are 1 initial, $2-2.6 \mathrm{Krad}, 3-7.6 \mathrm{Krad}, 4-18.2$ Krad, 5 - $769 \mathrm{Krad}$. Dose rate was $120 \mathrm{rad} / \mathrm{hr}$ for doses 2 through 4 and $3.5 \times 10^{4} \mathrm{rad} / \mathrm{hr}$ for dose 5 . Cumulative doses for 768 are 1 - initial, 2 - 2.4 Krad, 3 - 11 Krad, 4 - 20 Krad, 5 - 834 Krad. Dose rate for dose 2 through 4 was 132 $\mathrm{rad} / \mathrm{hr}$ and $3.6 \times 10^{4}$ for dose 5 .

wavelength was found. Figure 2 shows the spectra measured for the two crystals after correcting for phototube quantum efficiency and monochromator grating efficiency. The radioluminescence peaks at around $515 \mathrm{~nm}$ for sample 767 , while sample 768 shows a lower peak at around $486 \mathrm{~nm}$. Although the photoluminescence appears to peak at longer wavelengths $(527 \mathrm{~nm}$ for 767 and $513 \mathrm{~nm}$ for 768 ), at least part of this shift can be attributed to self absorbance in the crystal.

The decay time of the scintillation emission was measured for a number of samples using the single photon counting technique [6]. The resulting spectra were fit to the sum of three exponentials of the form $S_{1} / \tau_{1} \cdot e^{-t / \tau_{1}}+$ $S_{2} / \tau_{2} \cdot e^{-t / \tau_{2}}+S_{3} / \tau_{3} \cdot e^{-t / \tau_{3}}$. Figure 3 shows the spectrum measured for sample 768 , which gave values $\tau_{1}=2.1$ $\mathrm{ns}, \tau_{2}=13.0 \mathrm{~ns}$ and $\tau_{3}=348 \mathrm{~ns}$ for the decay time constants with amplitudes $S_{1}=.10, S_{2}=.08$ and $S_{3}=.82$.
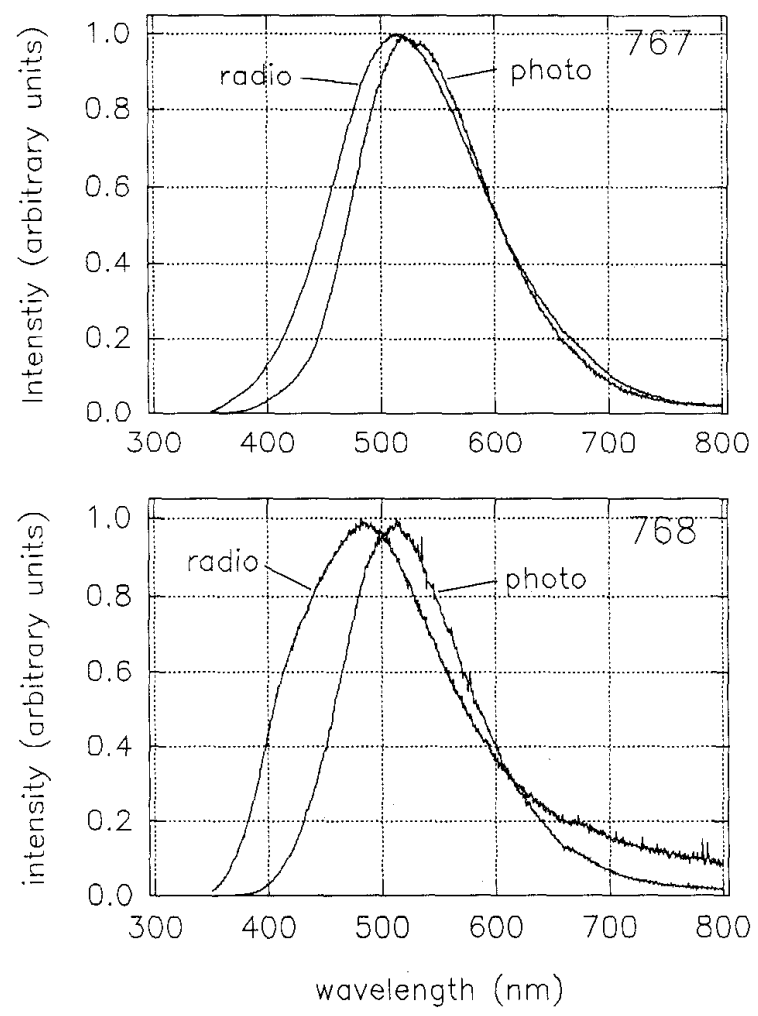

Figure 2: Radioluminescence and photoluminescence emission spectra for samples 767 and 768. All spectra have been corrected for phototube quantum efficiency and monochromator efficiency and normalized to one at their peak.

In general, all samples showed this same three component spectrum with approximately the same decay times, namely, a very fast component in the range from 2-5 ns, a medium fast component on the range from 10-20 ns, and a slow component in the range from 300-500 ns. It should be noted that, while the fast components constitute a substantial fraction of the total light output, the integrated light yield of the slow component is much greater than the combined fast components.

Both samples were irradiated using ${ }^{60} \mathrm{Co}$ gamma rays up to a maximum total dose of 769 krad for sample 767 and $834 \mathrm{krad}$ for sample 768 . In both cases, the initial irradiation was carried out at a low dose rate $(\sim 120-130 \mathrm{rad} / \mathrm{hr})$ in order to study any rapid changes in either sample. As shown in Figure 1, sample 768 damaged quickly, exhibiting a series of strong, broad absorption bands in the region between 400 and 700 $\mathrm{nm}$, and continued to damage with increasing dose. This damage was similar to that which was observed in undoped samples [4]. On the other hand, sample 767 showed very little damage, both at the initial low dose rate, and at the higher dose rate up to the maximum dose. Other samples showed levels of damage which were in between samples 767 and 768 . 


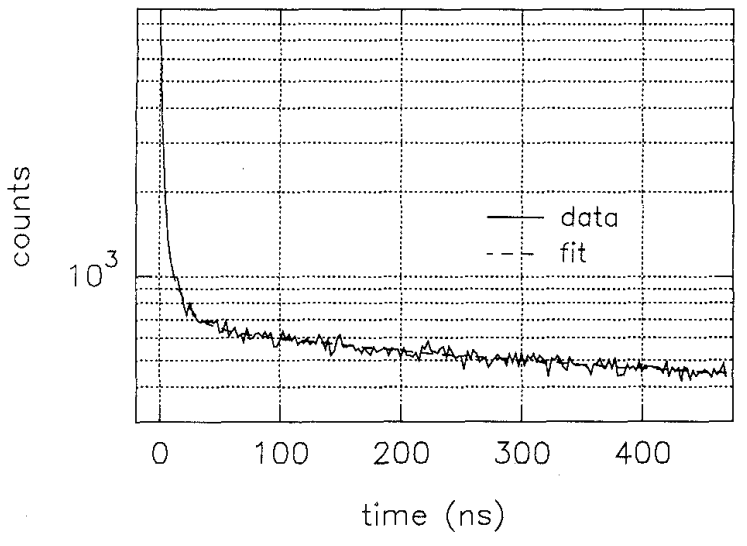

Figure 3: Decay time of the scintillation emission for sample 768. A fit to the spectrum gives three components with times constants of 2.1 ns, $13.0 \mathrm{~ns}$ and $348 \mathrm{~ns}$ (see text).

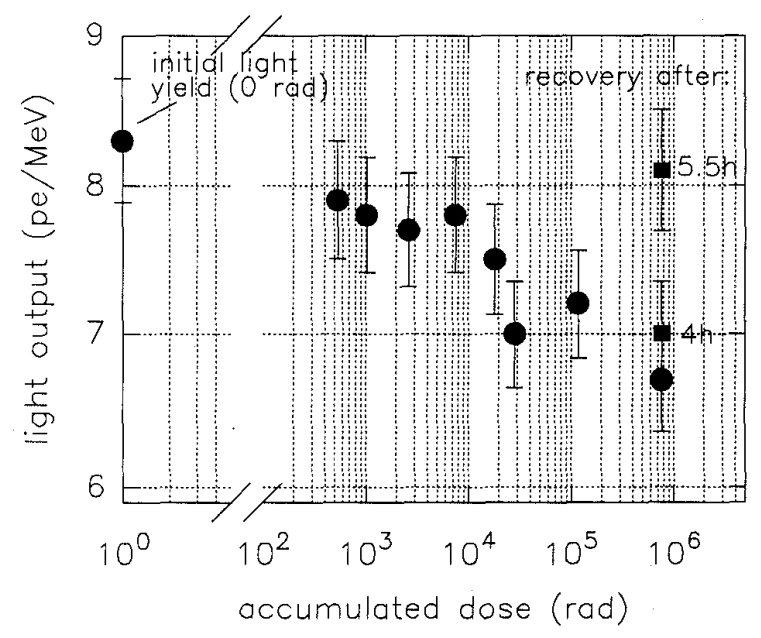

Figure 4: Light output vs accumulated dose for sample 767. Except where indicated, all points were measured approximately $30 \mathrm{~min}$ utes after irradiation.

The light output of samples 767 and 768 was measured before irradiation using a 2" dia. phototube (Hamamatsu R2059) and a ${ }^{60} \mathrm{Co}$ source. The photoelectron yield was determined from the measured position of the photopeak and the known gain of the phototube in ADC channels per photoelectron. Both crystals gave a yield of $\sim$ 8 p.e./ $\mathrm{MeV}$, which was approximately independent of position along the crystal. After the initial dose, the light output from sample 768 was not measurable due to the increased self absorbance and interference from radiation induced phosphorescence. However, the light output from sample 767 was measurable even up to the maximum dose, as shown in Figure 4. The light output, measured approximately 30 minutes after the final dose, showed a

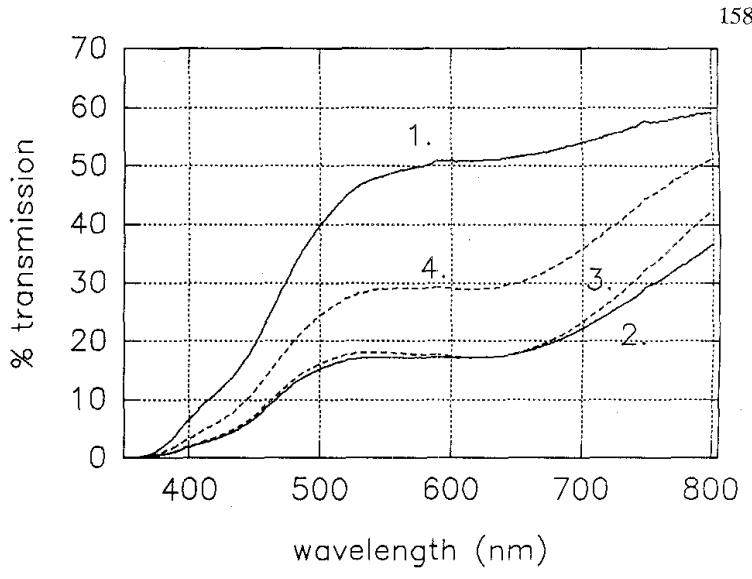

Figure 5: Room temperature recovery for sample 768 kept in the dark. 1- initial, 2- 30 min after 1 Mrad, 3- 4 days recovery, 4- 18 days recovery.

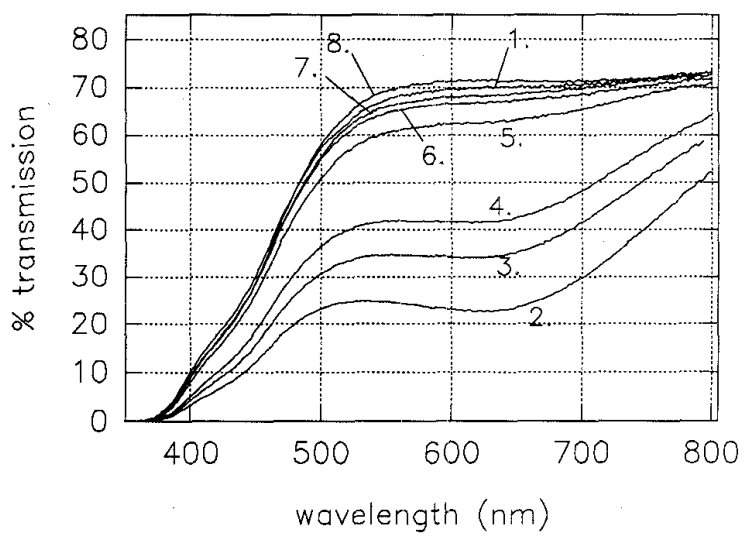

Figure 6: Effect of optical bleaching on sample 768. Longitudinal transmission curves correspond to: 1- initial; 2- immediately after a total dose of $834 \mathrm{krad}$; optical bleaching: $3-5$ hours at $700 \mathrm{~nm} ; 4-12$ hours at $700 \mathrm{~nm}$; 55 hours at $600 \mathrm{~nm} ; 6-10$ hours at $600 \mathrm{~nm}$; 7- 7 hours at $640 \mathrm{~nm} ; 8$ - 2 hours of thermal annealing at $200^{\circ} \mathrm{C}$.

slight decrease of about $15 \%$, but recovered to its original value within 5.5 hours.

Sample 768 damaged much more than 767, but showed spontaneous recovery in its transmission when kept in the dark at room temperature, as shown in Figure 5. Other samples which damaged showed a similar behavior with a recovery time characterized by a time constant of $\sim 160$ hours. Recovery in sample 768 could also be induced by optical bleaching using light in the $600-700 \mathrm{~nm}$ range, as shown in Figure 6 . Several hours of bleaching with $700 \mathrm{~nm}$ light produced significant recovery when applied immediately after irradiation. Further recovery was induced by several additional hours of bleaching with $600 \mathrm{~nm}$ and $640 \mathrm{~nm}$ light, but a certain level of 
1588

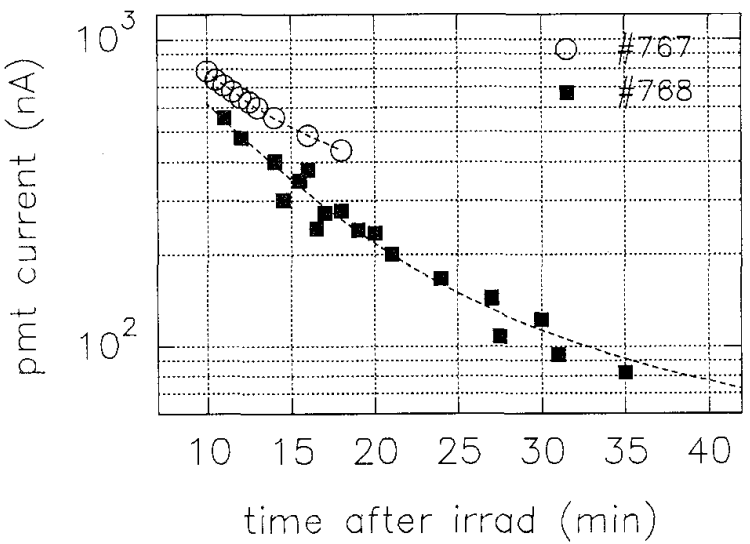

Figure 7: Phosphorescence intensity vs time for samples 767 and 768 after gamma ray doses of 0.7 and $1.0 \mathrm{Mrad}$, resp.

permanent damage remained. However, after bleaching, thermal annealing for two hours at a temperature of 200 ${ }^{\circ} \mathrm{C}$ produced more recovery, and resulted in a transmission which was actually slightly higher than its original value.

Figure 7 shows the radiation induced phosphorescence in samples 767 and 768 as a function of time starting approximately 10 minutes after gamma ray doses of 0.7 and 1.0 Mrad, respectively. The apparent lower phosphorescence intensity in sample 768 was at least partly due to its higher induced absorption. The magnitude of the phosphorescence intensity was rather weak in both samples, which made it difficult to determine the actual emission spectrum. For lower radiation doses applied at lower dose rates, as indicated in Figure 1, the phosphorescence intensity measured immediately after irradiation corresponded to only $\sim 0.1$ photoelectrons per nanosecond. However, using a series of edge cutoff filters with higher dose exposures, it was possible to determine that the phosphorescence emission peaks at $\sim 500 \mathrm{~nm}$, which is approximately the same wavelength as the radioluminescence and photoluminescence. The time constant for the phosphorescence decay, as given by fitting the data shown in Figure 7, was 6.0 minutes for sample 767 and 6.7 minutes for samples 768 . However, measurements made on the phosphorescence starting within a few seconds after irradiation showed a more intense, rapidly decaying component which decayed away by more than two orders of magnitude within approximately 1 minute.

Both samples also exhibited a weak thermoluminescence (TL), as shown in Figure 8. The thermoluminescence glow curves were obtained by exposing roughly $10 \mathrm{mg}$ of each crystal to 1 Mrad of ${ }^{60} \mathrm{Co}$ gamma rays. The TL was then measured 10 minutes after irradiation from $40^{\circ} \mathrm{C}$ to $400{ }^{\circ} \mathrm{C}$ at a heating rate of $2{ }^{\circ} \mathrm{C} / \mathrm{sec}$. The resulting curves show that sample 768 has a stronger TL response than 767 , implying a higher concentration of charge traps and luminescence centers. However, the TL signal in both samples is rather weak, and is roughly 3 or 4 orders

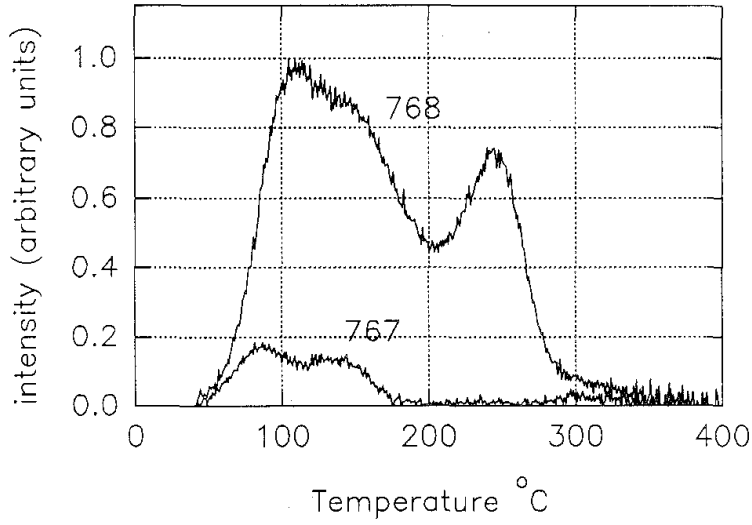

Figure 8: Thermoluminescence glow curves for samples 767 and 768 .

of magnitude less in intensity than a typical sample of $\mathrm{BaF}_{2}$. The longer lived phosphorescence observed in both crystals most likely arises from the two lowest temperature glow peaks. Sample 768 has at least 3 peaks at 110,150 , and $245^{\circ} \mathrm{C}$, and sample 767 has at least 2 peaks at 90 and $140^{\circ} \mathrm{C}$. These data indicate that the lowest temperature trap has a depth of about $0.7 \mathrm{eV}$ for both crystals, but to estimate the trap depths for the other peaks would require additional measurements.

\begin{tabular}{|c|c|c|}
\hline Element & \multicolumn{2}{|c|}{ Sample concentration (ppm) } \\
\hline Sample & 767 & 768 \\
\hline $\mathrm{Na}$ & 9.6 & 1.8 \\
$\mathrm{Si}$ & 44.0 & 52.0 \\
$\mathrm{Ca}$ & 1.0 & 2.5 \\
$\mathrm{Nb}$ & 8.1 & 7.1 \\
$\mathrm{Mo}$ & 17.0 & 15.0 \\
\hline
\end{tabular}

Table 1. GDMS analysis for samples 767 and 768

A trace element analysis was performed on both samples using Particle Induced X-ray Emission (PIXE) and Glow Discharge Mass Spectroscopy (GDMS) in order to determine the stoichiometry of the crystals and to search for trace element impurities [7]. The results showed that both crystals were essentially stoichiometric $\mathrm{PbWO}_{4}$, although there was an indication that the $\mathrm{W} / \mathrm{Pb}$ ratio varied by $\sim 5 \%$ from one end of the crystal to the other. A survey of 77 elements showed similar levels of impurities in the few ppm to sub ppm level in both samples. Table 1 lists the impurities detected in a portion of each sample, taken 3-5 $\mathrm{mm}$ below the surface of the crystal, at a level greater than $1 \mathrm{ppm}$. The lighter elements ( $\mathrm{Na}, \mathrm{Si}$ and Ca) could be present due to certain materials used in the growth or cutting and polishing processes. The niobium levels of 7-8 ppm indicate that a substantial fraction of the niobium which was added to the initial melt (30 ppm) was lost during growth. 
Finally, it is interesting to note the high level of molybdenum in both samples. The presence of molybdenum has recently been shown to be a possible cause of phosphorescence in some samples [8]. Due to the long decay time of the phosphorescence, this emission may contribute to the apparent total light yield of crystals with high molybdenum content when measured in a dc mode, as opposed to a single photon counting mode. It is therefore of considerable interest to study the effect of molybdenum and other impurities in greater detail, and to try and correlate their presence with other properties of the crystals. However, given that two samples which exhibit very different degrees of radiation hardness have rather similar levels of trace element impurities, it appears that the radiation damage properties may determined by other effects, such as structural differences or defects.

\section{CONCLUSIONS}

A study of a number of lead tungstate crystals has revealed a large sample to sample variation in many of their properties. This may be due to variations in the growth process or raw materials, which could be improved with a better understanding of how these factors influence the various properties of the crystals. The optical transmission varies considerably, and some samples show sizable regions of visible inhomogeneities. The radioluminescence spectra also vary from crystal to crystal, with the peak occurring in the $480-520 \mathrm{~nm}$ range. The scintillation decay time shows two major components in the 2-3 ns and 15-20 ns range, along with a third slow component in the several hundred nanosecond range. Some samples, which do not necessarily show the best optical transmission before irradiation, exhibit particularly good radiation hardness. Initial trace element analyses carried out on samples having very different characteristics have shown similar levels of trace element impurities, indicating that the observed differences may be due to other effects, such as structural differences or defects. Further work is under way to attempt to correlate these results with the important performace properties of the crystals.

\section{REFERENCES}

[1] A.Fyodorov et.al., Further Progress in Lead Tungstate Crystals, LAAP-EXP94.24, Dec. 1994.

[2] P.Lecoq et.al., Lead Tungstate $\left(\mathrm{PbWO}_{4}\right)$ Scintillators for LHC EM-Calorimetry, CERN/PEE, CMS Technical Note 94-308.

[3] Samples obtained through the Institute for Nuclear Problems, Minsk, Belarus, and IHEP Protvino, Russia.

[4] J.Kierstead et.al., Proceedings of the Symposium on Scintillator and Phosphor Materials, Materials Research Society, Vol. 348 (1994) 475.
[5] P.W.Levy, J. Phys. Chem. Solids 52 (1991) 319-349.

[6] L.M.Bollinger and G.E.Thomas, Rev. Sci. Inst. 32 (1961) 1044.

[7] PIXE and GDMS analyses were performed by Charles Evans \& Associates, Redwood City, CA.

[8] M.Kobayashi et.al., Further Studies on ExcitationEmission Spectra, Radiation Damage and Mechanical Properties of $\mathrm{PbWO}_{4}$, Proceedings of the SCINT95 International Conference on Inorganic Scintillators, Delft, The Netherlands, Aug.28-Sep.1, 1995, Delft University Press, Ed. P. Dorenbos and C.W.E. van. Eijk. 